\title{
Acknowledgement to Referees
}

(c) Springer Nature Switzerland AG 2021

Dear Reader,

Welcome to the final issue of the American Journal of Clinical Dermatology for 2021.

I wish to reflect on this year's achievements, and to thank all those who have contributed their time and effort to guarantee the quality of the content published in the journal.

The high quality of content published in the American Journal of Clinical Dermatology has been reflected in the most recent Impact Factor of 7.403 and CiteScore ${ }^{\mathrm{TM}}$ of 9.6. These place the journal fifth and third, respectively, in the dermatology categories. Further, the American Journal of Clinical Dermatology has published content in a timely manner, with an average time from submission to acceptance of 78 days.

In 2021, over 70 articles have been published. The most popular of these in terms of downloads from SpringerLink have been:

Erythema Nodosum: A Practical Approach and Diagnostic Algorithm

Effects of Diet on Acne and Its Response to Treatment

Rosacea: New Concepts in Classification and Treatment

Use of IL-23 Inhibitors for the Treatment of Plaque Psoriasis and Psoriatic Arthritis: A Comprehensive Review

Dermatitis Herpetiformis: An Update on Diagnosis and Management

Extended Safety Analysis of Baricitinib $2 \mathrm{mg}$ in Adult Patients with Atopic Dermatitis: An Integrated Analysis from

Eight Randomized Clinical Trials

Conjunctivitis in Dupilumab Clinical Trials for Adolescents with Atopic Dermatitis or Asthma

Effect of Dupilumab on Laboratory Parameters in Adolescents with Atopic Dermatitis: Results from a Randomized, Placebo-Controlled, Phase 3 Clinical Trial

Pharmacokinetics of Ruxolitinib in Patients with Atopic Dermatitis Treated With Ruxolitinib Cream: Data from Phase II and III Studies

Pooled Results of Two Randomized Phase III Trials Evaluating VP-102, a Drug-Device Combination Product Containing

Cantharidin $0.7 \%$ (w/v) for the Treatment of Molluscum Contagiosum

Social media sharing and other online mentions of information are other metrics with which to measure the reach of articles. This year the following articles published in the American Journal of Clinical Dermatology scored highly on the Altmetric system:

The PROVe Study: US Real-World Experience with Chlormethine/Mechlorethamine Gel in Combination with Other Therapies for Patients with Mycosis Fungoides Cutaneous T-Cell Lymphoma

Biologic Treatment Algorithms for Moderate-to-Severe Psoriasis with Comorbid Conditions and Special Populations: A Review

Extended Safety Analysis of Baricitinib $2 \mathrm{mg}$ in Adult Patients with Atopic Dermatitis: An Integrated Analysis from Eight Randomized Clinical Trials

Vulvar Melanoma: Molecular Characteristics, Diagnosis, Surgical Management, and Medical Treatment

Erythema Nodosum: A Practical Approach and Diagnostic Algorithm 
Integrated Safety Analysis of Abrocitinib for the Treatment of Moderate-to-Severe Atopic Dermatitis From the Phase II and Phase III Clinical Trial Program

Coronavirus Disease 2019 (COVID-19)-Associated Hospitalization and Mortality in Patients with Psoriasis: A Population-Based Study

Recommendations for Vaccination in Children with Atopic Dermatitis Treated with Dupilumab: A Consensus Meeting, 2020

Nail Psoriasis and Quality-of-Life Measurement in Clinical Trials: Call for the Use of Nail-Specific Instruments

The COVID-19 pandemic has continued to present many challenges and I would like to thank all who have contributed to ensuring the journal has thrived despite the ongoing issues.

I would like to start by thanking the authors of the articles published in the American Journal of Clinical Dermatology over the course of 2021. The enthusiasm of all authors for their chosen fields and their willingness to contribute content to the journal are crucial for its continued success.

The quality of published articles is also testament to the diligence of the peer reviewers. I would like to acknowledge the following individuals who acted as reviewers for the American Journal of Clinical Dermatology in the last 12 months:

Masashi Akiyama, Japan

Jill I. Allbritton, USA

Emiliano Antiga, Italy

Lisa Arkin, USA

Victor Asensi, Spain

Anna Balato, Italy

Federico Bardazzi, Italy

Johann W. Bauer, Austria

Luigi Bennardo, Italy

Ramesh Bhat, India

Neal Bhatia, USA

Andreas Bircher, Switzerland

Joke A. Bouwstra, the Netherlands

Kanwaljit Brar, USA

Valeria Brazzelli, Italy

Martina Burlando, Italy

Valerie D. Callender, USA

Scott Cameron, Canada

Anna Campanati, Italy

Carla Carnovale, Italy

Wei-Ti Chen, Taiwan, Republic of China

WenChieh Chen, Germany

Yi-Hsing Chen, Taiwan, Republic of China

Pavel V. Chernyshov, Ukraine

Hsien-Yi Chiu, Taiwan, Republic of China

Jaelim Cho, Republic of Korea

Ellie Choi, Singapore

Raj Chovatiya, USA

Catherine Chung, USA

Wen-Hung Chung, Taiwan, Republic of China

Curtis Cole, USA

Claudio Conforti, Italy

Pippa Corrie, UK

Martha Council, USA
Jeffrey J. Crowley, USA

Giovanni Damiani, Italy

William Damsky, USA

Steven Daveluy, USA

Dawn Davis, USA

Marjolein S. de Bruin-Weller, The Netherlands

Vito Di Lernia, Italy

Brittany Dulmage, USA

Steven R. Feldman, USA

Francesca L. Filon, Italy

Alan Fleischer, USA

Regina Folster-Holst, Germany

Peter C. Friedman, USA

Hideki Fujita, Japan

Amit Garg, USA

Yolanda Gilaberte, Spain

Brian Ginsberg, USA

Roberta Giuffrida, Italy

Michael H. Gold, USA

Melinda Gooderham, Canada

Emmy Graber, USA

Ayman Grada, USA

Sonia Gran, UK

Sungat Grewal, USA

Emmanuella Guenova, Switzerland

Lauren Guggina, USA

Catherine A. Harwood, UK

Gulen Hatemi, Turkey

Courtney E. Heron, USA

Mohammad Jafferany, USA

Benjamin Kaffenberger, USA

Ayse Serap Karadag, Turkey

Sarolta Karpati, Hungary

Subuhi Kaul, USA 
Reiji Kojima, Japan

Taro Kono, Japan

Piotr Krajewski, Poland

Shawn G. Kwatra, USA

Francesco Lacarrubba, Italy

Joseph Lam, Canada

Julien Lambert, Belgium

Abigail Langton, UK

Christopher Lao, USA

Ding-Dar Lee, Taiwan, Republic of China

Monica K. Li, Canada

Michael D. Linder, Norway

Ivan Litvinov, Canada

Woraphong Manuskiatti, Thailand

Michael A. Marchetti, USA

Paul Massey, USA

Matteo Megna, Italy

Laurent Misery, France

Hideaki Miyachi, Japan

Juan Arcadio Molina-Guarneros, Mexico

Elisa Molinelli, Italy

Rotraut Mössner, Germany

Ladan Mostaghimi-Tehrani, USA

Jenny E. Murase, USA

Tarun Narang, India

Ken Natsuga, Japan

Reinhard Neubert, Germany

Yuta Norimatsu, Japan

Grace Obeid, Lebanon

Robert Ofenloch, Germany

Sang Ho Oh, Republic of Korea

Yukari Okubo, Japan

Peck Y. Ong, USA

Nahide Onsun, Turkey

Jart A.F. Oosterhaven, The Netherlands

Christina Oprica, Sweden

Ashley O'Toole, Canada

Evangelia Papadavid, Greece

Lawrence C. Parish, USA

Wiebke Peitsch, Germany

José-Juan Pereyra-Rodriguez, Spain

Sara Perkins, USA

Mauro Picardo, Italy

Miriam K. Pomeranz, USA

Caitlin Purvis, USA

Varun K. Ranpariya, USA

Adam Reich, Poland

Hans C. Ring, Denmark

David Rosmarin, USA
Angelo Ruggiero, Italy

Ricardo Ruiz-Villaverde, Spain

Gerardo Russo, USA

Piotr Rutkowski, Poland

Jorge Santos-Juanes, Spain

Christopher J. Sayed, USA

Enno Schmidt, Germany

Marcus Schmitt-Egenolf, Sweden

Robert A. Schwartz, USA

Jakob Schwensen, Denmark

Maryanne M. Senna, USA

Vivian Y. Shi, USA

Tetsuo Shiohara, Japan

Pietro Sollena, Italy

Teresa Song, USA

Alexander Sorokin, USA

Reinhart Speeckaert, Belgium

Jonathan M. Spergel, USA

Julia Spoendlin, Switzerland

Georg Stary, Austria

Luca Stingeni, Italy

Ake Svensson, Sweden

Yayoi Tada, Japan

Charles Taieb, France

Marina Talamonti, Italy

Ryoji Tanei, Japan

Emil A. Tanghetti, USA

Amy F. Taub, USA

Kim Thomas, UK

Jakub Tolar, USA

Nataša Toplak, Slovenia

Rochelle R. Torgerson, USA

Tiago Torres, Portugal

Anne Marie Tremaine, USA

Ya-Chu Tsai, Taiwan, Republic of China

Stephen K. Tyring, USA

Peter C.M. van de Kerkhof, The Netherlands

Jan A.M. van Laar, The Netherlands

Katerina Vavrova, Czech Republic

Ron Vender, Canada

Sarah Walsh, UK

Jenny Zhuoyue Wang, USA

Guy F. Webster, USA

Jonathan S. Weiss, USA

Bin Yang, China

Jensen Yeung, Canada

Xuejun Zhang, China

Jiafang Zhu, China

Christos C. Zouboulis, Germany 
I am also very grateful to the members of the journal's Honorary Editorial Board, who have acted as peer reviewers and authors, and have provided guidance on journal content, policy, and processes.

Dietrich Abeck, Group Practice for Dermatology and Allergology, Munich, Germany

Tina S. Alster, Washington Institute of Dermatologic Laser Surgery, Washington DC, USA

Emily M. Altman, Summit Medical Group, Berkeley Heights, NJ, USA

Kyle T. Amber, Rush University Medical Center, Chicago, IL, USA

Klaus E. Andersen, Odense University Hospital, Odense, Denmark

Enzo Berardesca, University of Miami, Miami, FL, USA

Jose-Manuel Carrascosa, Hospital Universitari Germans Trias i Pujol, Badalona, Barcelona, Spain

Clay J. Cockerell, University of Texas Southwestern Medical Center, Dallas, TX, USA

Philip R. Cohen, University of California San Diego, San Diego, CA, USA

Scott A. Davis, University of North Carolina, Chapel Hill, NC, USA

Robert P. Dellavalle, University of Colorado School of Medicine, Aurora, CO, USA

Brigitte Dréno, Nantes University, Nantes, France

Madeleine Duvic, University of Texas, Houston, TX, USA

Peter Elsner, Friedrich Schiller University, Jena, Germany

Joseph C. English III, University of Pittsburgh, Pittsburgh, PA, USA

Vincent Falanga, Boston University School of Medicine, Boston, MA, USA

Alice B. Gottlieb, New York Medical College, Valhalla, NY, USA

Christopher E.M. Griffiths, University of Manchester, Manchester, UK

Aditya K. Gupta, Mediprobe Research Inc., London, ON, Canada

C. William Hanke, Laser and Skin Surgery Center of Indiana, Carmel, IN, USA

Rod J. Hay, International Foundation for Dermatology, London, UK

Amor Khachemoune, Veterans Affairs Medical Center, Brooklyn, NY, USA

Mark G. Lebwohl, The Icahn School of Medicine at Mount Sinai, New York, NY, USA

Howard I. Maibach, University of California San Francisco, San Francisco, CA, USA

Andrew G. Messenger, Royal Hallamshire Hospital, Sheffield, UK

Daniel Mimouni, Tel Aviv University, Tel Aviv, Israel

Mariya Miteva, University of Miami L. Miller School of Medicine, Miami, FL, USA

Ulrich Mrowietz, University Medical Center of Schleswig-Holstein, Kiel, Germany

Amy S. Paller, Northwestern University Feinberg School of Medicine, Chicago, IL, USA

Tania J. Phillips, Boston University School of Medicine, Boston, MA, USA

Neil Shear, University of Toronto, Toronto, ON, Canada

Thierry Simonart, Private Practice, Brussels, Belgium

Keiran S. Smalley, Moffitt Cancer Center \& Research Institute, Tampa, FL, USA

Tsen-Fang Tsai, National Taiwan University Hospital, Taipei, Taiwan, Republic of China

Jeffrey M. Weinberg, Mount Sinai St. Luke's and Mount Sinai Beth Israel, New York, NY, USA

Uwe Wollina, Academic Teaching Hospital Dresden-Friedrichstadt, Dresden, Germany

Springer Nature has continued to support the global response to COVID-19 by making all relevant content immediately and freely available. Indeed, the American Journal of Clinical Dermatology has published several papers relevant to the COVID-19 pandemic this year that are free to access, including:

Coronavirus Disease 2019 (COVID-19)-Associated Hospitalization and Mortality in Patients with Psoriasis: A Population-Based Study

Teledermatology During COVID-19: An Updated Review

Biologic Treatment Algorithms for Moderate-to-Severe Psoriasis with Comorbid Conditions and Special Populations: A Review

In terms of other important company initiatives, the Springer Nature 'Sustainable Development Goals (SDG) Programme' is aiming to connect researchers who are tackling the world's toughest challenges with practitioners in policy and business, while the 'Women in Science' initiative aims to help empower more women scientists to achieve scientific excellence. Finally, 
I am delighted to inform you that Springer Nature is now carbon neutral for its direct operations (offices, fleet, and flights) and earlier this year signed The Climate Pledge, making a commitment to be Net Zero carbon by 2040.

Returning to the American Journal of Clinical Dermatology, the editorial program for 2022 is well underway, and I am looking forward to bringing you many high-quality and authoritative articles in the field of dermatology over the coming year.

I thank you for your continued support.

With best wishes,

Kathy Fraser, Editor 